\title{
A NONCOMMUTATIVE GENERALIZATION OF AUSLANDER'S LAST THEOREM
}

\author{
EDGAR E. ENOCHS, OVERTOUN M. G. JENDA, AND J. A. LÓPEZ-RAMOS
}

Received 26 July 2004

We show that every finitely generated left $R$-module in the Auslander class over an $n$ perfect ring $R$ having a dualizing module and admitting a Matlis dualizing module has a Gorenstein projective cover.

In 1966 [1], Auslander introduced a class of finitely generated modules having a certain complete resolution by projective modules. Then using these modules, he defined the G-dimension ( $\mathrm{G}$ ostensibly for Gorenstein) of finitely generated modules. It seems appropriate then to call the modules of G-dimension 0 the Gorenstein projective modules. In [4], Gorenstein projective modules (whether finitely generated or not) were defined. In the same paper, the dual notion of a Gorenstein projective module was defined and so a relative theory of Gorenstein modules was initiated (cf. $[2,5]$ and references therein). In [12], Grothendieck introduced the notion of a dualizing complex. A dualizing module for $R$ is one whose deleted injective resolution is a dualizing complex. Then a local Noetherian ring $R$ is Gorenstein if and only if $R$ is itself a dualizing module for $R$. In this case, Auslander announced the result that over such a ring, every finitely generated module has a finitely generated Gorenstein projective cover (or equivalently, a minimal maximal Cohen-Macaulay approximation). In [9], this result was generalized to the situation where $R$ is a local Cohen-Macaulay ring having a dualizing module. More recently, in [13], Jørgensen has shown the existence of Gorenstein projective precovers for every module over a commutative Noetherian ring with a dualizing complex. Using Christensen [3], we here introduce the notion of a dualizing bimodule associated with a pair of Noetherian rings (but not necessarily commutative ones). In [6], it was shown that in this situation, every module in the Auslander class defined by the pair of rings admits a Gorenstein projective precover. Now we give examples where the dualizing bimodule has a double structure over the same noncommutative Noetherian ring and that in this case, if the ring also admits a Matlis dualizing module, (cf. [8] or [10]), we particularize the result to the existence of a stronger approximation, that is, every finitely generated module in the Auslander class has a finitely generated Gorenstein projective cover.

Given a class of $R$-modules $\mathscr{F}$, an $\mathscr{F}$-precover of a left $R$-module $M$ is a morphism $F \stackrel{\varphi}{\rightarrow} M$ with $F \in \mathscr{F}$ and such that if $F^{\prime} \stackrel{f}{\rightarrow} M$ is a morphism with $F^{\prime} \in \mathscr{F}$, then there is 
a morphism $F^{\prime} \stackrel{g}{\rightarrow} F$ such that $\varphi g=f$. If whenever $F=F^{\prime}$ and $f=\varphi$, then $g$ is always an

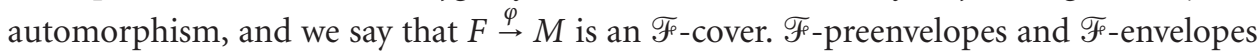
are defined dually.

A left $R$-module $M$ is said to be Gorenstein projective if there is an exact sequence

$$
\cdots \longrightarrow P_{1} \longrightarrow P_{0} \longrightarrow P^{0} \longrightarrow P^{1} \longrightarrow \cdots
$$

of projective left $R$-modules which remains exact whenever $\operatorname{Hom}_{R}(-, P)$ is applied to it for every projective module $P$ and such that $M=\operatorname{Ker}\left(P^{0} \rightarrow P^{1}\right)$. Gorenstein injectives are defined dually (cf. [5]).

Definition 1 [6, Definition 2.1]. Let $R$ be a right and left Noetherian ringand let ${ }_{R} V_{R}$ be an $R-R$-bimodule such that $\operatorname{End}\left({ }_{R} V\right)=R$ and $\operatorname{End}\left(V_{R}\right)=R$. Then $V$ is said to be a dualizing module if it satisfies the following three conditions:

(i) $\operatorname{id}\left({ }_{R} V\right) \leq r$ and id $\left(V_{R}\right) \leq r$ for some integer $r$,

(ii) $\operatorname{Ext}_{R}^{i}\left({ }_{R} V,{ }_{R} V\right)=\operatorname{Ext}_{R}^{i}\left(V_{R}, V_{R}\right)=0$ for all $i \geq 1$,

(iii) ${ }_{R} V$ and $V_{R}$ are finitely generated.

The preceding definition is given in [6] for a bimodule ${ }_{S} V_{R}$, where $S$ and $R$ are left and right Noetherian rings, respectively, but through this paper, we will consider the case $S=R$.

Examples. If $R$ is a Cohen-Macaulay local ring of Krull dimension $d$ admitting a dualizing module $\Omega$ (see [7]), then $\Omega$ is a dualizing module in this sense.

If $R$ is an $n$-Gorenstein ring (cf. [5, Definition 9.1.9]), then ${ }_{R} R_{R}$ is a dualizing module.

Let $R=\oplus_{g \in G} R_{g}$ be a strongly graded ring over a finite group $G$, right and left Noetherian and let $R_{e} V_{R_{e}}$ be a dualizing module (for $R_{e}, e \in G$ is the neutral element in $G$ ). Then $W=R \otimes_{R_{e}} V \otimes_{R_{e}} R$ is a dualizing module (for $R$ ).

Let $R$ be right and left Noetherian and let ${ }_{R} V_{R}$ be a dualizing module. Then $R[[x]] V[[x]]_{R[[x]]}$ is a dualizing module.

In [11], the authors defined Auslander and Bass classes of modules over a CohenMacaulay ring admitting a dualizing module. We now use the bimodule $V$ to introduce the corresponding classes in a noncommutative setting.

Definition 2. Let $R$ be right and left Noetherian and let ${ }_{R} V_{R}$ be a dualizing module. Define the left Auslander class $\mathscr{A}^{l}(R)$ (relative to $V$ ) as those left $R$-modules $M$ such that $\operatorname{Tor}_{i}^{R}(V, M)=0$ and $\operatorname{Ext}_{R}^{i}\left(V, V \otimes_{R} M\right)=0$ for all $i \geq 1$ and such that the natural morphism $M \rightarrow \operatorname{Hom}_{R}\left(V, V \otimes_{R} M\right)$ is an isomorphism. The right Auslander class $\mathscr{A}^{r}(R)$ is the class of right $R$-modules $M$ such that $\operatorname{Tor}_{i}^{R}(M, V)=0$ and $\operatorname{Ext}_{R}^{i}\left(V, M \otimes_{R} V\right)=0$ for all $i \geq 1$ and such that the natural morphism $M \rightarrow \operatorname{Hom}_{R}\left(V, M \otimes_{R} V\right)$ is an isomorphism.

The left Bass class $\mathscr{B}^{l}(R)$ (relative to $V$ ) is defined as those left $R$-modules $N$ such that $\operatorname{Ext}_{R}^{i}(V, N)=0$ and $\operatorname{Tor}_{i}^{R}\left(V, \operatorname{Hom}_{R}(V, N)\right)=0$ for all $i \geq 1$ and such that the natural morphism $V \otimes_{R} \operatorname{Hom}_{R}(V, N) \rightarrow N$ is an isomorphism. The right Bass class $\mathscr{B}^{r}(R)$ is defined as those right $R$-modules $N$ such that $\operatorname{Ext}_{R}^{i}(V, N)=0$ and $\operatorname{Tor}_{i}^{R}\left(\operatorname{Hom}_{R}(V, N), V\right)=0$ for all $i \geq 1$ and such that the natural morphism $\operatorname{Hom}_{R}(V, N) \otimes_{R} V \rightarrow N$ is an isomorphism.

We recall the following definition from [8]. 
Definition 3. A ring $R$ has a Matlis dualizing module if there is an $(R, R)$-bimodule $E$ such that ${ }_{R} E$ and $E_{R}$ are both injective cogenerators and such that the canonical maps $R \rightarrow \operatorname{Hom}_{R}\left({ }_{R} E_{R},{ }_{R} E_{R}\right)$ and $R \rightarrow \operatorname{Hom}_{R}\left(E_{R}, E_{R}\right)$ are both bijections. $E$ will be called a Matlis dualizing module for $R$.

Several examples of Matlis dualizing modules are given in [8]. We now give some additional examples.

Examples. If $R$ is left and right Noetherian having a Matlis dualizing module $E$, then $E\left[x^{-1}\right]$ is a Matlis dualizing module for $R[[x]]$.

If $R$ is a strongly graded ring over a finite group, right and left Noetherian, and ${ }_{R_{e}} E_{R_{e}}$ is a dualizing module (for $R_{e}$ ), then $W=R \otimes_{R_{e}} E \otimes_{R_{e}} R$ is a dualizing module (for $R$ ).

In what follows, $R$ will always be a right and left Noetherian ring and if $E$ is a Matlis dualizing module for $R$, we will denote $M^{\vee}=\operatorname{Hom}_{R}(M, E)$ for $M \in R$-Mod or $M \in \operatorname{Mod}-R$.

Proposition 4. Let $R$ be a ring and let $V$ and $E$ be a dualizing module and a Matlis dualizing module for $R$, respectively. If $M \in R-M o d$ is finitely generated, then $M \in \mathscr{A}^{l}(R)$ if and only if $M^{\vee} \in \mathscr{B}^{r}(R)$.

Proof. Suppose that $M \in \mathscr{A}^{l}(R)$. Since $\operatorname{Tor}_{i}^{R}(V, M)=0$, then

$$
\operatorname{Ext}_{R}^{i}\left(V, M^{\vee}\right) \cong\left(\operatorname{Tor}_{i}^{R}(V, M)\right)^{\vee}=0 \quad \forall i \geq 1 .
$$

On the other hand, $\left(\operatorname{Tor}_{i}^{R}\left(\operatorname{Hom}_{R}\left(V, M^{\vee}\right), V\right)\right)^{\vee} \cong \operatorname{Ext}_{R}^{i}\left(V,\left(\operatorname{Hom}_{R}\left(V, M^{\vee}\right)\right)^{\vee}\right)$. But $\left(\operatorname{Hom}_{R}\left(V, M^{\vee}\right)\right)^{\vee}=\operatorname{Hom}_{R}\left(\operatorname{Hom}_{R}\left(V, \operatorname{Hom}_{R}(M, E)\right), E\right) \cong \operatorname{Hom}_{R}\left(\operatorname{Hom}_{R}\left(V \otimes_{R} M, E\right)\right.$, $E) \cong\left(V \otimes_{R} M\right)^{v v}$ and since $V \otimes_{R} M$ is finitely generated, $\left(V \otimes_{R} M\right)^{\vee \vee} \cong V \otimes_{R} M$ (cf. [8]), and so we get that

$$
\operatorname{Ext}_{R}^{i}\left(V,\left(\operatorname{Hom}_{R}\left(V, M^{\vee}\right)\right)^{\vee}\right) \cong \operatorname{Ext}_{R}^{i}\left(V, V \otimes_{R} M\right)=0 \quad \forall i \geq 1 .
$$

Therefore, $\operatorname{Tor}_{i}\left(\operatorname{Hom}_{R}\left(V, M^{\vee}\right), V\right)=0$ for all $i \geq 1$.

Finally, by hypothesis $M \cong \operatorname{Hom}_{R}\left(V, V \otimes_{R} M\right)$ and so $\operatorname{Hom}_{R}\left(V, V \otimes_{R} M\right)^{\vee} \cong M^{\vee}$ is an isomorphism. We also know that $\operatorname{Hom}_{R}\left(V, M^{\vee}\right) \otimes_{R} V \cong\left(V \otimes_{R} M\right)^{\vee} \otimes_{R} V$. Therefore, we only have to show that $\left(V \otimes_{R} M\right)^{\vee} \otimes_{R} V \rightarrow \operatorname{Hom}_{R}\left(V, V \otimes_{R} M\right)^{\vee}$ is an isomorphism to get that $\operatorname{Hom}_{R}\left(V, M^{\vee}\right) \otimes_{R} V \cong M^{\vee}$.

The functors $\left(V \otimes_{R} M\right)^{\vee} \otimes_{R}-$ and $\operatorname{Hom}_{R}\left(-, V \otimes_{R} M\right)^{\vee}$ are both right exact and the natural morphism

$$
\left(V \otimes_{R} M\right)^{\vee} \otimes_{R} R^{n} \longrightarrow \operatorname{Hom}_{R}\left(R^{n}, V \otimes_{R} M\right)^{\vee}
$$

is an isomorphism, and so the morphism is also an isomorphism for finitely generated modules, in particular for $V$.

Conversely, let now $N=M^{\vee}$ and suppose that $N \in \mathscr{B}^{r}(R)$. Since $M$ is finitely generated, we get that $N^{\vee} \cong M$. Now $\operatorname{Tor}_{i}^{R}(V, M)^{\vee} \cong \operatorname{Ext}_{R}^{i}\left(V, M^{\vee}\right)=\operatorname{Ext}_{R}^{i}(V, N)=0$ for all $i \geq 1$ and so $\operatorname{Tor}_{i}^{R}(V, M)=0$ for all $i \geq 1$.

Moreover, $\operatorname{Tor}_{i}^{R}\left(\operatorname{Hom}_{R}(V, N), V\right)=0$ for all $i \geq 1$ and so

$$
0=\operatorname{Tor}_{i}^{R}\left(\operatorname{Hom}_{R}(V, N), V\right)^{\vee} \cong \operatorname{Ext}_{R}^{i}\left(V, \operatorname{Hom}_{R}(V, N)^{\vee}\right) .
$$


But $\operatorname{Hom}_{R}(V, N)^{\vee}=\operatorname{Hom}_{R}\left(V, M^{\vee}\right)^{\vee} \cong\left(V \otimes_{R} M\right)^{\vee \vee} \cong V \otimes_{R} M$, and therefore $\operatorname{Ext}_{R}^{i}(V$, $\left.V \otimes_{R} M\right)=0$ for all $i \geq 1$. It only remains to show that $M \rightarrow \operatorname{Hom}_{R}\left(V, V \otimes_{R} M\right)$ is an isomorphism.

Since $N^{\vee} \in \mathscr{B}^{r}(R)$, then $\operatorname{Hom}_{R}(V, N) \otimes_{R} V \rightarrow N$ is an isomorphism, and therefore

$$
N^{\vee} \cong\left(\operatorname{Hom}_{R}(V, N) \otimes_{R} V\right)^{\vee} \cong \operatorname{Hom}_{R}\left(V, \operatorname{Hom}_{R}(V, N)^{\vee}\right) .
$$

Then consider the natural transformation

$$
-\otimes_{R} \operatorname{Hom}_{R}(N, E) \longrightarrow \operatorname{Hom}_{R}\left(\operatorname{Hom}_{R}(-, N), E\right)
$$

This gives an isomorphism for $R^{n}$ and since both functors are right exact, it follows that $V \otimes_{R} \operatorname{Hom}_{R}(N, E) \cong \operatorname{Hom}_{R}\left(\operatorname{Hom}_{R}(V, N), E\right)$ and so

$$
M=N^{\vee} \longrightarrow \operatorname{Hom}_{R}\left(V, \operatorname{Hom}_{R}(V, N)^{\vee}\right) \cong \operatorname{Hom}_{R}\left(V, V \otimes_{R} M\right)
$$

is an isomorphism.

We now recall from [6] that a ring $R$ is said to be left (right) $n$-perfect if every left (right) flat $R$-module has projective dimension less than or equal to $n$.

Left perfect rings, commutative Noetherian rings of finite Krull dimension, the universal enveloping algebra $U(g)$ of a Lie algebra of dimension $n$, and $n$-Gorenstein rings are all examples of left $n$-perfect rings. Also, if $R$ is left $n$-perfect, then $R[x], R[[x]]$, the crossed product $R * U(g)$, and the Weyl algebra $A_{k}(R)$ are left $k$-perfect for some $k$ (cf. [6]).

Proposition 5. Let $R$ be a right and left $n$-perfect ring, let $V$ and $E$ be a dualizing module of finite left and right injective dimension $r$ and a Matlis dualizing module for $R$, respectively, and let $G \in R$-Mod be finitely generated. Then $G$ is Gorenstein projective if and only if $G^{\vee}$ is Gorenstein injective.

Proof. If $G$ is Gorenstein projective, by [5, Proposition 10.2.6], there exists an exact sequence

$$
0 \longrightarrow G \longrightarrow P_{r+n} \longrightarrow \cdots \longrightarrow P_{0} \longrightarrow M \longrightarrow 0
$$

where every $P_{i}$ is a finitely generated projective. Now since $G$ and $P_{i}, i=0, \ldots, r+n$, are in $\mathscr{A}^{l}(R)$ by [6, Proposition 2.3], then $M \in \mathscr{A}^{l}(R)$ by [6, Proposition 2.7]. Now, $P_{i}^{\vee}$ is injective for every $i=0, \ldots, r+n$ and by the preceding proposition $M^{\vee} \in \mathscr{B}^{r}(R)$, so by [6, Theorem 2.11], $G^{\vee}$ is Gorenstein injective.

Conversely, let $G^{\vee}$ be Gorenstein injective. Since $G$ is finitely generated, there exists a flat preenvelope $G \rightarrow F$ which factors via a finitely generated free module $R^{k}$, so we can assume that $F$ is finitely generated free. But then, since $R^{\vee}=E$, we get that $E^{n} \rightarrow G^{\vee}$ is an injective precover, and so the injective cover of $G^{\vee}$ is Artinian. Then there is an exact sequence in Mod- $R$,

$$
0 \longrightarrow N \longrightarrow E_{r-1} \longrightarrow \cdots \longrightarrow E_{0} \longrightarrow G^{\vee} \longrightarrow 0
$$


where every $E_{i}$ is Artinian and injective. Then $N \in \mathscr{B}^{r}(R)$ since $G^{\vee}$ and so are $E_{i}, i=$ $0, \ldots, r-1$. In this way, we see that

$$
0 \longrightarrow G^{\vee \vee}=G \longrightarrow E_{0}^{\vee} \longrightarrow \cdots \longrightarrow E_{r-1}^{\vee} \longrightarrow N^{\vee} \longrightarrow 0
$$

is exact with $N^{\vee} \in \mathscr{A}^{l}(R)$ and $E_{i}^{\vee}$ is projective for every $i=0, \ldots, r-1$ and therefore by $[6$, Theorem 2.14], $G$ is Gorenstein projective.

The following result appears in [6] but we include a proof here for completeness.

Theorem 6. Let $R$ be a left $n$-perfect ring and ${ }_{R} V_{R}$ a dualizing module for $R$ such that $\operatorname{id}\left({ }_{R} V\right), \operatorname{id}\left(V_{R}\right) \leq r$. If $M \in \mathscr{A}^{l}(R)$, then it has a Gorenstein projective precover $G \stackrel{\varphi}{\rightarrow} M \rightarrow 0$ such that $\operatorname{pd}(\operatorname{Ker}(\varphi)) \leq r-1$.

Proof. Let $0 \rightarrow C \rightarrow F_{r-1} \rightarrow \cdots \rightarrow F_{0} \rightarrow M \rightarrow 0$ be a (partial) projective resolution of $M$. Then, by [6, Lemma 2.12], $C$ is Gorenstein projective. Now let

$$
\cdots P_{1} \longrightarrow P_{0} \longrightarrow P^{0} \longrightarrow P^{1} \longrightarrow \cdots
$$

be an exact sequence of projective modules such that $C=\operatorname{Ker}\left(P^{0} \rightarrow P^{1}\right)$ and it remains exact whenever $\operatorname{Hom}_{R}(-, P)$ is applied for every projective $P$. We consider

$$
0 \longrightarrow P^{0} \longrightarrow \cdots \longrightarrow P^{r-1} \longrightarrow D \longrightarrow 0
$$

exact. Then we have a commutative diagram:

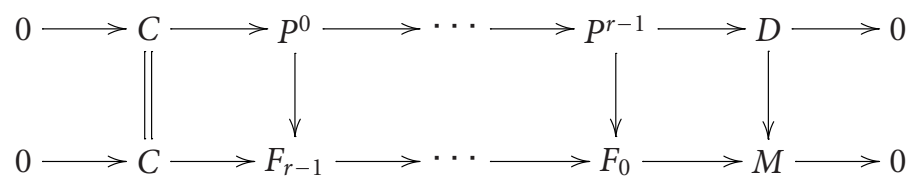

The associated complex to this diagram (i.e., the mapping complex)

$$
0 \longrightarrow C \longrightarrow C \oplus P^{0} \longrightarrow \cdots \longrightarrow F_{0} \oplus D \longrightarrow M \longrightarrow 0
$$

is exact and has as a subcomplex the exact sequence $0 \rightarrow C \rightarrow C \rightarrow 0$. Then quotient complex

$$
0 \longrightarrow P^{0} \longrightarrow \cdots \longrightarrow F_{0} \oplus D \longrightarrow M \longrightarrow 0
$$

is exact and all of its terms are projective except perhaps $F_{0} \oplus D$. Now if $0 \rightarrow L \rightarrow F_{0} \oplus D \rightarrow$ $M \rightarrow 0$ is exact with $\operatorname{pd}(L)<\infty$, then $\operatorname{pd}(L) \leq r-1$. Since $F_{0} \oplus D$ is Gorenstein projective and $\operatorname{Ext}_{R}^{1}(X, L)=0$ for every Gorenstein projective $X$, it follows that $F_{0} \oplus D \rightarrow M$ is the desired precover.

Given a class $\mathscr{C}$ of $R$-modules, we let ${ }^{\perp} C$ be the class of $R$-modules $F$ such that $\operatorname{Ext}_{R}^{1}(F$, $C)=0$ for every $C \in \mathscr{C}$. We let $C^{\perp}$ be the class of $R$-modules $F$ such that $\operatorname{Ext}_{R}^{1}(C, F)=0$ for every $C \in \mathscr{C}$. A pair of classes of $R$-modules $(\mathscr{F}, \mathscr{C})$ is called a cotorsion theory if $\mathscr{F}^{\perp}=\mathscr{C}$ and $\perp \mathscr{C}=\mathscr{F}$. A cotorsion theory is said to be complete if for every $R$-module $M$, 
there is an exact sequence $0 \rightarrow M \rightarrow C \rightarrow F \rightarrow 0$ such that $C \in \mathscr{C}$ and $F \in \mathscr{F}$, or equivalently if there is an exact sequence $0 \rightarrow C \rightarrow F \rightarrow M \rightarrow 0$ such that $C \in \mathscr{C}$ and $F \in \mathscr{F}$, which is equivalent to say that every $R$-module has a special $\mathscr{F}$-precover and a special $\mathscr{C}$-preenvelope (cf. [5]). A cotorsion theory is said to be perfect if every $R$-module has an $\mathscr{F}$-cover and a $\mathscr{C}$-envelope.

Now since $R$ is left Noetherian, then $\operatorname{Hom}(-,-)$ is left balanced by $\operatorname{Inj} \times \operatorname{Inj}$ on $R$ Mod $\times R$-Mod, and therefore we can compute left derived functors of $\operatorname{Hom}_{R}(-,-)$ using left injective resolutions in the second variable constructed with injective covers or right injective resolutions in the first one (cf. [5, Example 8.3.5]). We will denote them by $\operatorname{Ext}_{i}(-,-) i \geq 0$ and $\overline{\operatorname{Ext}}^{0}(M, N)$, and $\overline{\operatorname{Ext}}_{0}(M, N)$ will denote the cokernel and the kernel of the natural morphism

$$
\operatorname{Ext}_{0}^{R}(M, N) \longrightarrow \operatorname{Hom}_{R}(M, N) .
$$

THeORem 7. Let $R$ be a right n-perfect ring and let ${ }_{R} V_{R}$ be a dualizing module for $R$ such that $\operatorname{id}\left({ }_{R} V\right), \operatorname{id}\left(V_{R}\right) \leq r$. If $\mathscr{L}$ and GorInj denote the classes of right $R$-modules of finite injective dimension and Gorenstein injective, then ( $\mathscr{L}$, GorInj) is a perfect cotorsion theory of $\mathscr{B}^{r}(R)$.

Proof. Suppose that $L \in \mathscr{B}^{r}(R) \cap{ }^{\perp}$ GorInj. Then $\operatorname{Ext}_{R}^{1}(L, G)=0$ for every $G$ Gorenstein injective. Now if $G$ is Gorenstein injective, then there exists an exact sequence $0 \rightarrow G^{\prime} \rightarrow$ $E_{0} \rightarrow G \rightarrow 0$ with $E_{0}$ injective and $G^{\prime}$ Gorenstein injective. By [5, Theorem 8.2.7], $\overline{\operatorname{Ext}}_{R}^{0}(L$, $G) \cong \operatorname{Ext}_{R}^{1}\left(L, G^{\prime}\right)=0$ for every Gorenstein injective $G$. Analogously, $\operatorname{Ext}_{1}^{R}(L, G) \cong \overline{\operatorname{Ext}}_{0}^{R}(L$, $\left.G^{\prime}\right)=0$ and by induction, $\operatorname{Ext}_{i}^{R}(L, G)=0$ for all $i \geq 1$ and for every Gorenstein injective $G$.

Now let $0 \rightarrow L \rightarrow E^{0} \rightarrow \cdots \rightarrow E^{r+n} \rightarrow C \rightarrow 0$ be a (partial) injective resolution of $L$. By [6, Lemma 2.9], $C$ is Gorenstein injective and so $\operatorname{Ext}_{n+r}^{R}(L, G)=0$ by the above. Therefore

$$
\operatorname{Hom}_{R}\left(E^{r+n+1}, C\right) \longrightarrow \operatorname{Hom}_{R}\left(E^{r+n}, C\right) \longrightarrow \operatorname{Hom}_{R}\left(E^{r+n-1}, C\right)
$$

is exact and so $C$ is a direct summand of $E^{r+n}$ which shows that $\operatorname{id}(L)<\infty$. If $L \in \mathscr{L}$, then it is immediate that $\operatorname{Ext}_{R}^{1}(L, G)=0$ for every Gorenstein injective $G$.

Suppose now that $G \in \mathscr{B}^{r}(R) \cap L^{\perp}$. Then by [6, Theorem 2.11], $G$ is Gorenstein injective. If $G$ is Gorenstein injective, then it is immediate that $G \in \mathscr{L}^{\perp}$.

Therefore $(\mathscr{L}$, GorInj) is a cotorsion theory. By [6, Theorem 2.16], it is complete. Finally, since $R$ is right Noetherian, $\mathscr{L}$ is closed under direct limits and so by [5, Theorem 7.2.6], ( $\mathscr{L}$, GorInj) is perfect.

THeOREM 8. Let $R$ be a left and right n-perfect ring admitting a Matlis dualizing module and let ${ }_{R} V_{R}$ be a dualizing module for $R$ such that $\mathrm{id}\left({ }_{R} V\right), \operatorname{id}\left(V_{R}\right) \leq r$. If $M \in \mathscr{A}^{l}(R)$ is finitely generated, then $M$ has a Gorenstein projective cover $G \stackrel{\varphi}{\rightarrow} M$ such that $G$ is finitely generated and $\operatorname{pd}(\operatorname{Ker}(\varphi)) \leq r-1$.

Proof. By Theorem 6, there is an exact sequence $0 \rightarrow L \rightarrow G \rightarrow M \rightarrow 0$ with $G$ Gorenstein projective and $\operatorname{pd}(L) \leq r-1$, which can be supposed finitely generated. Then if $0 \rightarrow$ $M^{\vee} \rightarrow G^{\vee} \rightarrow L^{\vee} \rightarrow 0$ is exact with $G^{\vee}$ Gorenstein injective by Proposition 5 and $\mathrm{id}\left(L^{\vee}\right)<$ $\infty$, therefore

$$
\operatorname{Hom}_{R}\left(G^{\vee}, N\right) \longrightarrow \operatorname{Hom}_{R}\left(M^{\vee}, N\right) \longrightarrow \operatorname{Ext}_{R}^{1}\left(L^{\vee}, N\right)=0
$$


is exact for every Gorenstein injective $N$, which gives that $M^{\vee} \rightarrow G^{\vee}$ is a Gorenstein injective preenvelope. By the preceding theorem, $M^{\vee}$ has a Gorenstein injective envelope $\varphi: M^{\vee} \rightarrow C$, which is a summand of $G^{\vee}$ and so $C$ is artinian. But $\operatorname{Coker}(\varphi)$ is also a direct summand of $L^{\vee}$ and so $\operatorname{id}(\operatorname{Coker}(\varphi)) \leq r-1$. Then $\operatorname{pd}\left((\operatorname{Coker}(\varphi))^{\vee}\right)<\infty$ and therefore we have an exact sequence

$$
0 \longrightarrow(\operatorname{Coker}(\varphi))^{\vee} \longrightarrow C^{\vee} \stackrel{\varphi^{\vee}}{\longrightarrow} M \longrightarrow 0
$$

where $C^{\vee}$ is Gorenstein projective by Proposition 5 and $\operatorname{pd}\left((\operatorname{Coker}(\varphi))^{\vee}\right) \leq r-1$. Since $\operatorname{pd}\left((\operatorname{Coker}(\varphi))^{\vee}\right)<\infty$, it follows that $C^{\vee} \stackrel{\varphi^{\vee}}{\rightarrow} M$ is a Gorenstein projective precover. Finally, $C^{\vee} \stackrel{\varphi^{\vee}}{\rightarrow} M$ is the desired cover since $C^{\vee}$ and $M$ are reflexive.

Corollary 9. Let $R$ and $M$ be as in the previous theorem and let $G \rightarrow M$ be a Gorenstein projective cover. Then $\operatorname{pd}(M)<\infty$ if and only if $G \rightarrow M$ is a projective cover.

Proof. Suppose that $\operatorname{pd}(M)<\infty$. Then $\operatorname{pd}(C)<\infty$ and let

$$
0 \longrightarrow P_{k} \longrightarrow \cdots \longrightarrow P_{0} \longrightarrow G \longrightarrow 0
$$

be a projective resolution of $G$. Since $\operatorname{Ext}_{R}^{1}(C, P)=0$ for every projective $P$, it follows that the sequence splits and so $G$ is projective. The converse is immediate.

Corollary 10. Let $R$ and $V$ be as in the previous theorem and let $M \in A^{l}(R)$ be finitely generated. Then the minimal Gorenstein projective resolution of $M$ is of the form

$$
0 \longrightarrow P_{k} \longrightarrow \cdots \longrightarrow P_{1} \longrightarrow G_{0} \longrightarrow M \longrightarrow 0
$$

where $P_{i}$ is projective for every $i=1, \ldots, k$ and $k \leq r$.

Proof. By [6, Corollary 2.13], $M \in \mathscr{A}^{r}(R)$ if and only if there is an exact sequence

$$
0 \longrightarrow G_{k} \longrightarrow \cdots \longrightarrow G_{0} \longrightarrow M \longrightarrow 0
$$

where every $G_{i}, i=0, \ldots, k$, is Gorenstein projective and $k \leq r$. Now the result follows from the preceding corollary.

\section{Acknowledgment}

The third author was supported by BFM2002-02717 Grant and Junta de Andalucía FQM 0211.

\section{References}

[1] M. Auslander, Anneaux de Gorenstein, et torsion en algèbre commutative, Séminaire d'Algèbre Commutative dirigé par Pierre Samuel, 1966/1967. Texte rédigé, d'après des exposés de Maurice Auslander, Marquerite Mangeney, Christian Peskine et Lucien Szpiro. École Normale Supérieure de Jeunes Filles, Secrétariat mathématique, Paris, 1967 (French).

[2] L. W. Christensen, Gorenstein Dimensions, Lecture Notes in Mathematics, vol. 1747, Springer, Berlin, 2000. 


\section{A generalization of Auslander's last theorem}

[3] Semi-dualizing complexes and their Auslander categories, Trans. Amer. Math. Soc. 353 (2001), no. 5, 1839-1883.

[4] E. E. Enochs and O. M. G. Jenda, Gorenstein injective and projective modules, Math. Z. 220 (1995), no. 4, 611-633.

[5] _ Relative Homological Algebra, De Gruyter Expositions in Mathematics, vol. 30, Walter de Gruyter, Berlin, 2000.

[6] E. E. Enochs, O. M. G. Jenda, and J. A. López-Ramos, Dualizing modules and n-perfect rings, Proc. Edinb. Math. Soc. (2) 48 (2005), no. 1, 75-90.

[7] E. E. Enochs, O. M. G. Jenda, and J. Z. Xu, Foxby duality and Gorenstein injective and projective modules, Trans. Amer. Math. Soc. 348 (1996), no. 8, 3223-3234.

[8] L Lifting group representations to maximal Cohen-Macaulay representations, J. Algebra 188 (1997), no. 1, 58-68.

[9] _ A generalization of Auslander's last theorem, Algebr. Represent. Theory 2 (1999), no. 3, 259-268.

[10] E. E. Enochs, J. A. López-Ramos, and B. Torrecillas, On Matlis dualizing modules, Int. J. Math. Math. Sci. 30 (2002), no. 11, 659-665.

[11] H.-B. Foxby, Gorenstein dimensions over Cohen-Macaulay rings, Proc. International Conference on Commutative Algebras (W. Bruns, ed.), Universitat Osnabrück, Osnabrück, 1994, pp. $59-63$.

[12] R. Hartshorne, Local Cohomology, Lecture Notes in Mathematics, no. 41, Springer, Berlin, 1967, a seminar given by A. Grothendieck, Harvard University, 1961.

[13] P. Jørgensen, The Gorenstein projective modules are precovering, preprint.

Edgar E. Enochs: Department of Mathematics, College of Arts and Sciences, University of Kentucky, Lexington, KY 40506-0027, USA

E-mail address: enochs@ms.uky.edu

Overtoun M. G. Jenda: Department of Mathematics and Statistics, College of Sciences and Mathematics, Auburn University, AL 36849-5310, USA

E-mail address: jendaov@auburn.edu

J. A. López-Ramos: Departamento de Álgebra y Análisis, Facultad de Ciencias Experimentales, Matemático, Universidad de Almería, 04120 Almería, Spain

E-mail address: jlopez@ual.es 


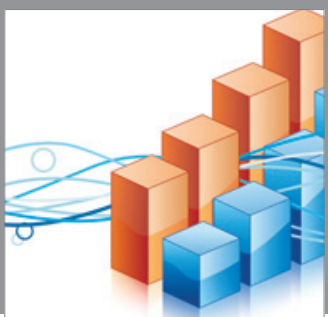

Advances in

Operations Research

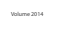

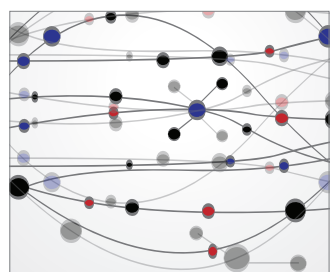

\section{The Scientific} World Journal
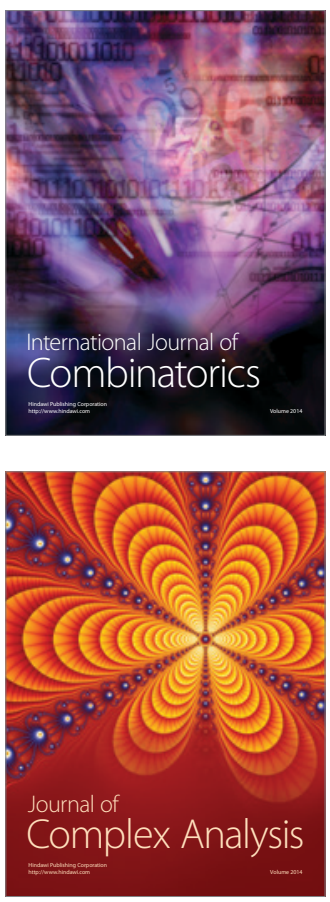

International Journal of

Mathematics and

Mathematical

Sciences
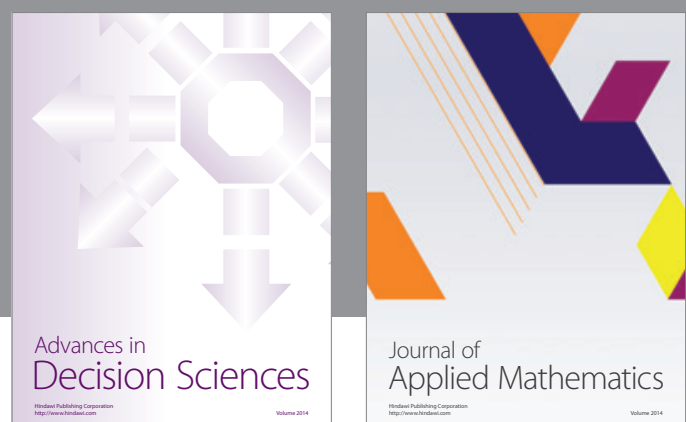

Journal of

Applied Mathematics
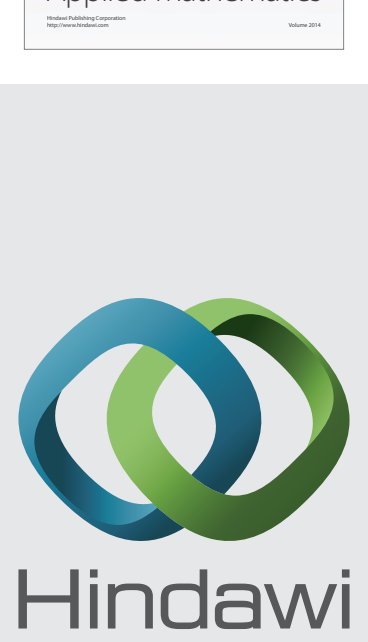

Submit your manuscripts at http://www.hindawi.com
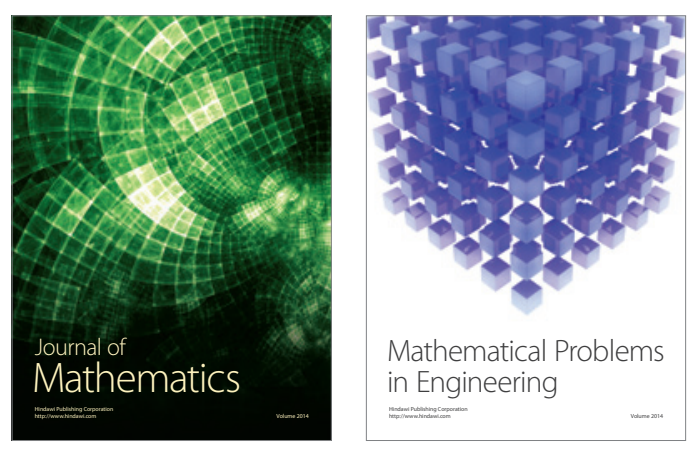

Mathematical Problems in Engineering
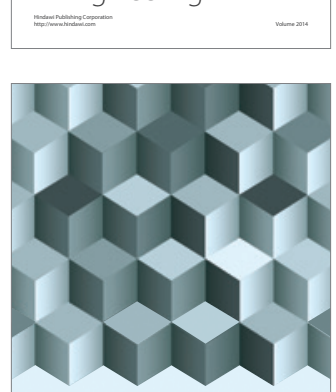

Journal of

Function Spaces
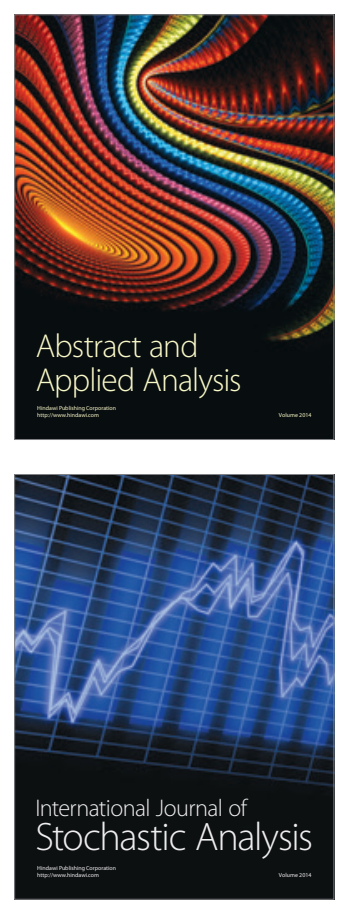

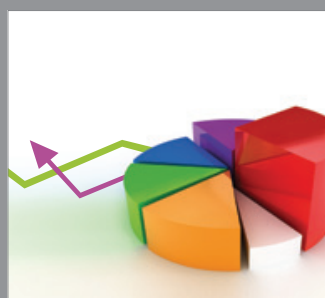

ournal of

Probability and Statistics

Promensencen
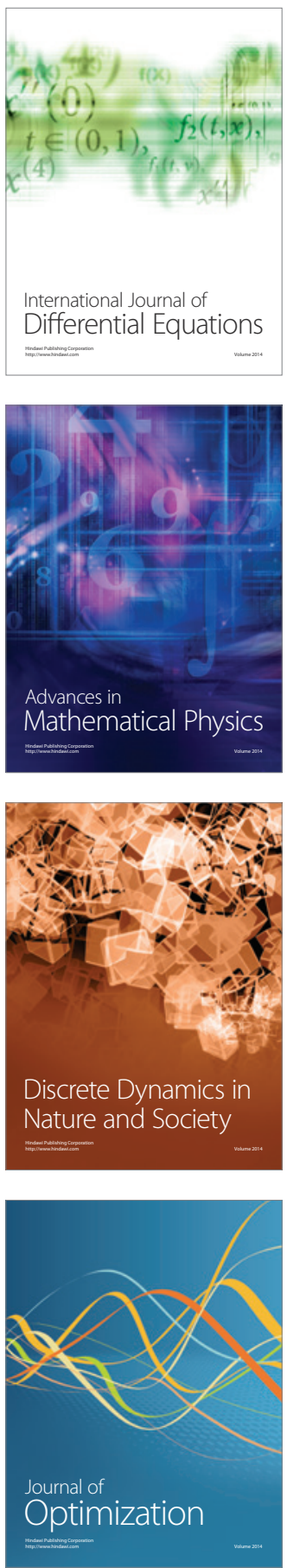